\title{
Ultra-performance liquid chromatography coupled with quadrupole time-of-flight mass spectrometry-based metabolic profiling of human serum prior to and following radical resection of colorectal carcinoma
}

\author{
LUFANG CHEN, CHUNXIA ZHANG, QIFENG GUI, YUE CHEN and YUNMEI YANG
}

\author{
Department of Geriatrics, The First Affiliated Hospital, School of Medicine, \\ Zhejiang University, Hangzhou, Zhejiang 310003, P.R. China
}

Received November 30, 2014; Accepted August 17, 2015

DOI: $10.3892 / \mathrm{mmr} .2015 .4289$

\begin{abstract}
Nearly one quarter of patients with colorectal carcinoma (CRC) were diagnosed at an advanced stage. Under these circumstances, radical resection of the tumor is the best strategy to enhance the five-year survival rate. However, up to $50 \%$ of post-operative patients experience cancer recurrence within the first few years. Therefore, post-operative surveillance is important. However, currently performed post-operative monitoring relies on relatively dated methods with insufficient sensitivity and specificity. The present study applied an advanced technology of ultra-performance liquid chromatography coupled with quadrupole time-of-flight mass spectrometry in order to examine changes in metabolite patterns in serum with the aim of identifying reliable biomarkers in patients with CRC at various time-points. Serum samples were collected from and $20 \mathrm{CRC}$ patients prior to radical resection (group 1) and one month following radical resection (group 2) as well as from 20 healthy volunteers (group 3). Multivariate pattern recognition was used to identify potential biomarkers of CRC. Compared with healthy volunteers, three groups of biomarkers were identified in patients with CRC $(\mathrm{P}<0.05)$, namely phosphatidylcholines (PCs), lysophosphatidylcholines (LPCs) and diacylglycerols (DAGs). However, no statistical difference in the levels of these biomarkers between pre-operative and post-operative $\mathrm{CRC}$ patients was identified $(\mathrm{P}>0.05)$. PCs and LPCs, which contain polyunsaturated fatty acids, were decreased, whereas LPCs and DAGs, which contain saturated fatty acids, were increased in CRC patients. The present
\end{abstract}

Correspondence to: Dr Yunmei Yang, Department of Geriatrics, The First Affiliated Hospital, School of Medicine, Zhejiang University, 79 Qingchun Road, Hangzhou, Zhejiang 310003, P.R. China E-mail: yangyunmei2014@hotmail.com

Key words: colorectal cancer, radical resection, metabonomics, ultra-performance liquid chromatography quadrupole time-of-flight mass spectrometry, supervised partial least-squares-latent structure discriminate analysis study demonstrated that obvious metabolic disturbances occur during the development of CRC and provided a novel analytic method, which is likely to be used as a diagnostic tool for CRC and may help to improve the patients' prognosis.

\section{Introduction}

Colorectal cancer (CRC) is the second leading cause of cancer-associated mortality (1). However, early diagnosis of CRC is challenging, as marked symptoms only appear in the period of disease progression. Therefore, most patients are diagnosed at a late stage (2). As a result, only radical resection of the tumor improves the clinical prognosis and provides a chance to prolong survival. However, up to 50\% of the post-operative patients suffer from a disease relapse within the first 3-5 years after operation (3). To reduce the risk of cancer recurrence as well as metastasis, post-operative patients must be closely monitored and their treatments adjusted to their condition. In general, post-operative assessments are based on clinical symptoms, serum biomarkers and imaging results (4). However, these relatively dated methods, including examination of serum tumor markers and computerized tomography, often lack sufficient sensitivity and specificity for guiding timely and appropriate therapies. Therefore, the development of more effective methods for the surveillance the disease process is required.

Small changes in the body can lead to large changes in metabolite levels (5). Metabonomics, one of the '-omics' technologies, provides quantitative measures to detect those large changes in the metabolic profiles of individuals responding to pathophysiological stimuli or genetic modification (6). Due to its high throughput, sensitivity and non-invasiveness, metabonomic analysis has already been used as A diagnostic tools in numerous human diseases, including diabetes (7), cancer (8), cardiovescular disease (9) and respiratory disease (10). Although a growing amount of research has provided experimental and clinical evidence for the close association between specific biomarkers and diagnosis of certain types of cancer, including CRC $(1,2,4)$, the application of metabonomic analysis to post-operative monitoring of CRC has rarely been reported (11). 
Ultra-performance liquid chromatography coupled with quadrupole time-of-flight mass spectrometry (UPLC-QTOF-MS) has been applied to evaluate the levels of certain disease-associated factors and biomarkers in biofluids which can be obtained by non-invasive techniques (including serum, urine or feces) as well as tissues (11). Due to the combination of high operating pressures and $1.7-\mu \mathrm{m}$ porous particles, UPLC is more sensitive and has a higher peak capacity and a better resolution compared with those of the traditional high performance liquid chromatography (HPLC) technique (12). Thus, this novel technique is considered to be suitable for large-scale untargeted metabonomics. The present study aimed to utilize UPLC-QTOF-MS for the post-operative monitoring of CRC patients by detecting changes in their serum metabolite patterns.

\section{Materials and methods}

Patients. All patients and healthy volunteers provided written informed consent in accordance with the institutional guidelines. The present study was approved by the Human Ethics Committee of the First Affiliated Hospital (School of Medicine, Zhejiang University, Hangzhou, China). Twenty patients with CRC who underwent radical resection, comprising nine cases of colon cancer and eleven cases of rectal cancer, as well as twenty age- and gender-matched healthy volunteers (individuals with no recorded tumors or other metabolic diseases) were recruited between March 2013 and August 2014 at the First Affiliated Hospital (School of Medicine, Zhejiang University, Hangzhou, China). Clinical and demographic characteristics of these individuals are listed in Table I.

The inclusion criteria were as follows: i) Positive diagnosis with stage A-C CRC according to the Duke classification (13); ii) histopathological confirmation of adenocarcinoma of the colon or rectum; iii) no prior enterectomy, radiotherapy and systemic chemotherapy; iv) no other metabolic diseases, such as diabetes mellitus; v) no inflammatory conditions. The exclusion criteria were as follows: i) Extra-abdominal metastasis or stage D Duke Classification; ii) concomitance of other malignant neoplasm; iii) application of any drugs in the previous two weeks; iv) drop-out during the study period.

Reagents and materials. HPLC-grade acetonitrile, leucine-enkephalin and formic acid were purchased from Sigma-Aldrich (St. Louis, MO, USA). Deionized water was produced using the Milli-Q ultrapure water purification system (Millipore, Bedford, MA, USA). The internal standards PC (17:0/0:0), PC (17:0/17:0), PE (17:0/17:0), PG (17:0/17:0), PS (17:0/17:0) and PA (17:0/17:0) were obtained from Avanti Polar Lipids Inc. (Alabaster, AL, USA), while DG (17:0/17:0/0:0), TG (17:0/17:0/17:0), MG (17:0/0:0/0:0), PC (16:1/0:0-D3), PC (16:1/16:1-D6) and TG (16:0/16:0/16:013C3) were obtained from Larodan Fine Chemicals (Malmo, Sweden).

Sample collection and preparation. Blood samples were obtained healthy controls and from CRC patients prior to radical resection as well as one month after surgery prior to any additional medical treatments, such as chemo-
Table I. Clinical and demographic data on healthy volunteers $(\mathrm{n}=20)$ and patients with CRC $(\mathrm{n}=20)$.

\begin{tabular}{lc}
\hline Characteristic & Value \\
\hline Healthy volunteers & \\
Mean age \pm SD (years) & $60.95 \pm 5.81$ \\
Age range (years) & $52-71$ \\
Gender (male/female) & $14 / 6$ \\
CRC patients & \\
Mean age \pm SD (years) & $61.10 \pm 7.55$ \\
Age range (years) & $51-73$ \\
Gender (male/female) & $14 / 6$ \\
Primary tumor site, $\mathrm{n}(\%)$ & \\
Colon & $9(45)$ \\
Rectum & $11(55)$ \\
Duke staging, $\mathrm{n}(\%)$ & \\
A & $1(5)$ \\
B & $5(25)$ \\
C & $14(70)$ \\
D & 0 \\
Differentiation grading, $\mathrm{n}(\%)$ & \\
Well & \\
Moderate & $14(70)$ \\
Poor & $6(30)$ \\
\hline
\end{tabular}

$\mathrm{SD}$, standard deviation; CRC, colorectal cancer.

therapy. All samples were collected from participants under fasting conditions. In all instances, $5 \mathrm{ml}$ blood from each proband was collected in tubes which did not contain any anti-coagulant substances. The collected blood was left on the laboratory bench for $30 \mathrm{~min}$ at room temperature prior to being centrifuged $\left(3,000 \mathrm{x} \mathrm{g}, 5 \mathrm{~min}, 4^{\circ} \mathrm{C}\right)$. The serum was carefully aspirated, transferred to a clean Eppendorf tube and subsequently stored at $-80^{\circ} \mathrm{C}$.

Prior to metabonomics analysis, the serum sample was thawed at room temperature. In order to precipitate the protein in serum sample, $200 \mu 1$ sample was added into $600 \mu \mathrm{l}$ ice-cold acetonitrile. The mixture was vortexed and centrifuged $\left(12,000 \mathrm{x} \mathrm{g}, 15 \mathrm{~min}, 4^{\circ} \mathrm{C}\right)$. Finally, the supernatant was transferred into a HPLC sample glass vial and stored at $4^{\circ} \mathrm{C}$ for UPLC-MS analysis. To evaluate the reproducibility and stability of the UPLC-MS system, $10 \mu 1$ of each sample was added into one vial to generate a pooled quality control (QC) sample, which was measured every eight samples throughout the experiment. The results of five consecutive runs of the QC sample are shown in Fig. 1; the stable retention times and tight overlap of the peaks demonstrate high repeatability and stability of the analytical system.

Blood biochemical parameter analysis. All blood samples were subjected to analysis using the Beckman-Coulter HMX automated system (Beckman-Coulter, Brea, CA, USA) to analyze the biochemical blood parameters, including hemoglobin ( $\mathrm{Hb})$, red blood cells (RBC) and white blood 
A

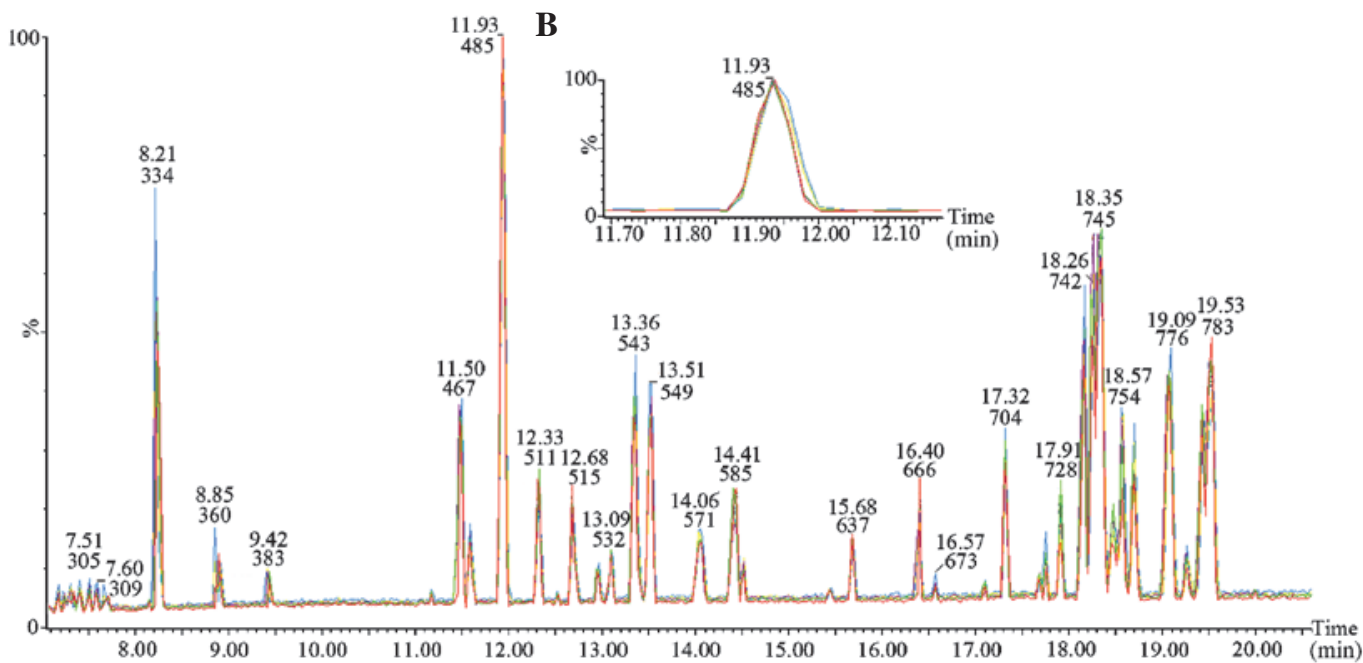

Figure 1. Analysis of quality control samples. (A) The five consecutive quality control chromatograms overlapped tightly, retention times were stable and no obvious drift was observed, indicating high stability and repeatability of the system. (B) Enlarged view of the eleventh minute. Each color represents one run. Numbers represent the retention time (top) and the peak integral (bottom).

cells (WBC). Serum carcinoembryonic antigen (CEA) was measured using a chemiluminescent technique (CEA Access; Beckman Coulter).

UPLC-QTOF-MS analysis. UPLC was performed using a Waters Acquity UPLC system (Waters Co., Milford, MA, USA) with a conditioned autosampler at $4^{\circ} \mathrm{C}$. A 5- $\mu$ l sample was injected into a Waters BEH C8 column (inner diameter $\mathrm{x}$ length, $2.1 \times 100 \mathrm{~mm} ; 1.7 \mu \mathrm{m}$ particle size), maintained at $50^{\circ} \mathrm{C}$. Mobile phase A contained $0.1 \%$ formic acid and $99.9 \%$ water or and mobile phase B contained $0.1 \%$ formic acid and $99.9 \%$ acetonitrile. The gradient elution program was as follows: $97 \% \mathrm{~A}$ in the initial 0-7 min, decreasing to $20 \% \mathrm{~A}$ at $8 \mathrm{~min}$ and to $2 \% \mathrm{~A}$ at $16 \mathrm{~min}$ at a flow rate of at $300 \mu \mathrm{l} / \mathrm{min}$. The conditions were kept constant for $5 \mathrm{~min}$ and then changed to $100 \% \mathrm{~B}$ within $50 \mathrm{sec}$, which was maintained for $3 \mathrm{~min}$. The column was equilibrated to $97 \%$ A over $25 \mathrm{~min}$, which was maintained for $5 \mathrm{~min}$.

Mass spectrometric analysis was performed using a Waters Q-TOF Premier mass spectrometer (Waters Co.) in positive electrospray ionization mode. The apparatus was previously calibrated using sodium formate (Sigma-Aldrich) and a lock mass of leucine enkephalin $(0.5 \mathrm{ng} / \mu \mathrm{l})$ was used for an accurate mass determination setting at $\mathrm{m} / \mathrm{z}, 556.2771 \mathrm{in}$ positive ion mode. The detection parameters were optimized as follows: Capillary voltage, $3 \mathrm{kV}$; and cone voltage, $40 \mathrm{~V}$. The scanning time was $0.3 \mathrm{sec}$ covering the $50-1,000$ Dalton mass range. The source temperature was set as $120^{\circ} \mathrm{C}$ and the desolvation gas temperature was $350^{\circ} \mathrm{C}$. Nitrogen (purchased as liquid nitrogen; Merriam-Graves Corp., White River Junction, VT, USA) was used as the nebulizer gas at a flow rate of $600 \mathrm{l} / \mathrm{h}$.

Data analysis. The UPLC-MS data collected in positive ion mode were pre-processed using Masslynx 4.1 software (Waters Co.). This application was used for peak alignment to obtain a list containing the $\mathrm{m} / \mathrm{z}$, retention time and intensities for all peaks detected. The pre-processed data were exported and analyzed using SIMCA-P+12.0 (Umetrics AB, Umea, Sweden). All data were normalized and Pareto scaled prior to multivariate statistical analysis. Unsupervised principal component analysis (PCA) was first used to separate treatment groups from the control group. If the separation between different groups was observed in the PCA plot, supervised partial least-squares latent structure discriminate analysis (PLS-DA) was then used to highlight the difference and obtain metabolites that contributed to the classification. Potential biomarkers were identified according to "variable of importance in projection' (VIP) values and S-plots.

To further identify the potential biomarkers, the Human Metabolome Database (HMDB; http://hmdb.ca/), PubChem compound (http://www.ncbi.nlm.nih. gov/) and Kyoto Encyclopedia of Genes and Genomes (KEGG, http://www. genome.jp/kegg/) databases were searched to match the selected ion spectra with those of the metabolites obtained from databases. MS analysis of the experimental samples in comparison with the standards was further performed for the validation of the potential biomarkers.

Statistical analysis. Blood biochemical parameters and serum metabolic biomarkers are expressed as the mean \pm standard deviation. The homogeneity of variances was verified using Bartlett's test. One-way analysis of variance with Bonferroni's post-hoc test was then performed to compare the spectral variables among different serum samples. $\mathrm{P} \leq 0.05$ was considered to indicate a statistically significant difference. Statistical analysis was performed using GraphPad Prism 5 (GraphPad Inc. La Jolla, CA, USA).

\section{Results}

Clinical biochemical features of CRC patients. The clinical biochemical serum parameters of patients prior to surgery (group 1), subsequent to surgery (group 2) and healthy controls (group 3) are presented in Fig. 2. Serum levels of CEA were significantly elevated in group 1 compared with 
A

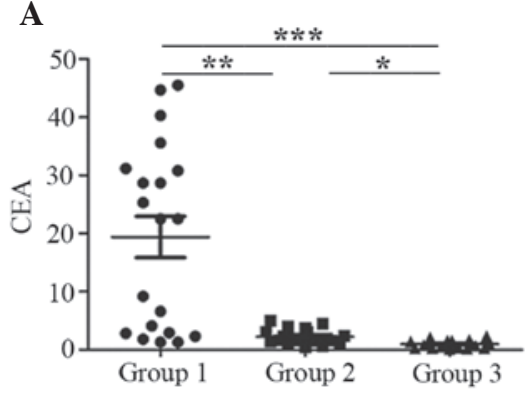

C

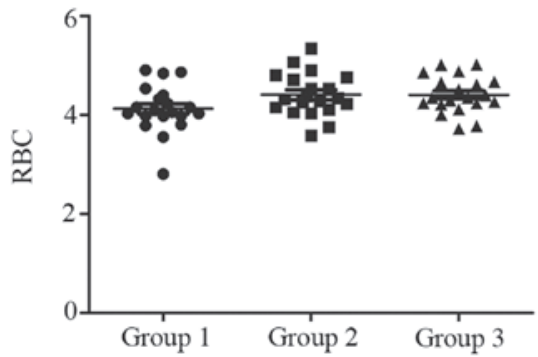

B

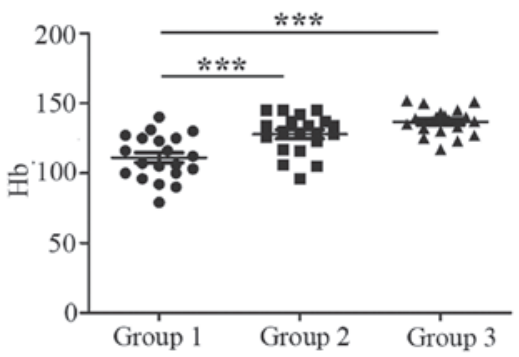

D

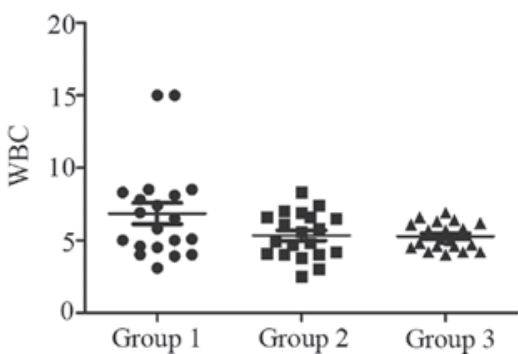

Figure 2. Clinical biochemical characteristics of CRC patients and healthy controls. The serum levels of (A) CEA (B) Hb (C) RBC (D) WBC in CRC patients prior and following operation and in healthy controls. The scatter diagrams show that serum levels of CEA in group 1 were markedly increased compared with those in group $3(\mathrm{P}<0.001)$. After the operation, CEA levels in group 2 decreased significantly $(\mathrm{P}<0.01)$ but remained higher than the levels in group 3 ( $\mathrm{P}<0.05)$. Unlike $\mathrm{CEA}$, following operation, serum levels of $\mathrm{Hb}$ in group 2 increased when compared to those in group 1 ( $\mathrm{P}<0.001)$, and no statistically significant difference was noted between group 2 and group 3. Furthermore, the RBC count was slightly lower and the WBC count was slightly higher in group 1 compared with that in groups 2 and 3, while RBC and WBC counts showed no statistically significant difference among the three groups (P>0.05). Each data point represents the value for one patient. Horizontal lines represent the mean values and bars represent the standard deviation. ${ }^{*} \mathrm{P}<0.05$, ${ }^{* *} \mathrm{P}<0.01,{ }^{* * *} \mathrm{P}<0.001 . \mathrm{Groups}$ : group $1, \mathrm{CRC}$ patients prior to operation; group 2, CRC patients posterior to operation; group 3, healthy controls. CEA, carcinoembryonic antigen; Hb, hemoglobin; RBC, red blood cell; WBC, white blood cell; CRC, colorectal cancer.

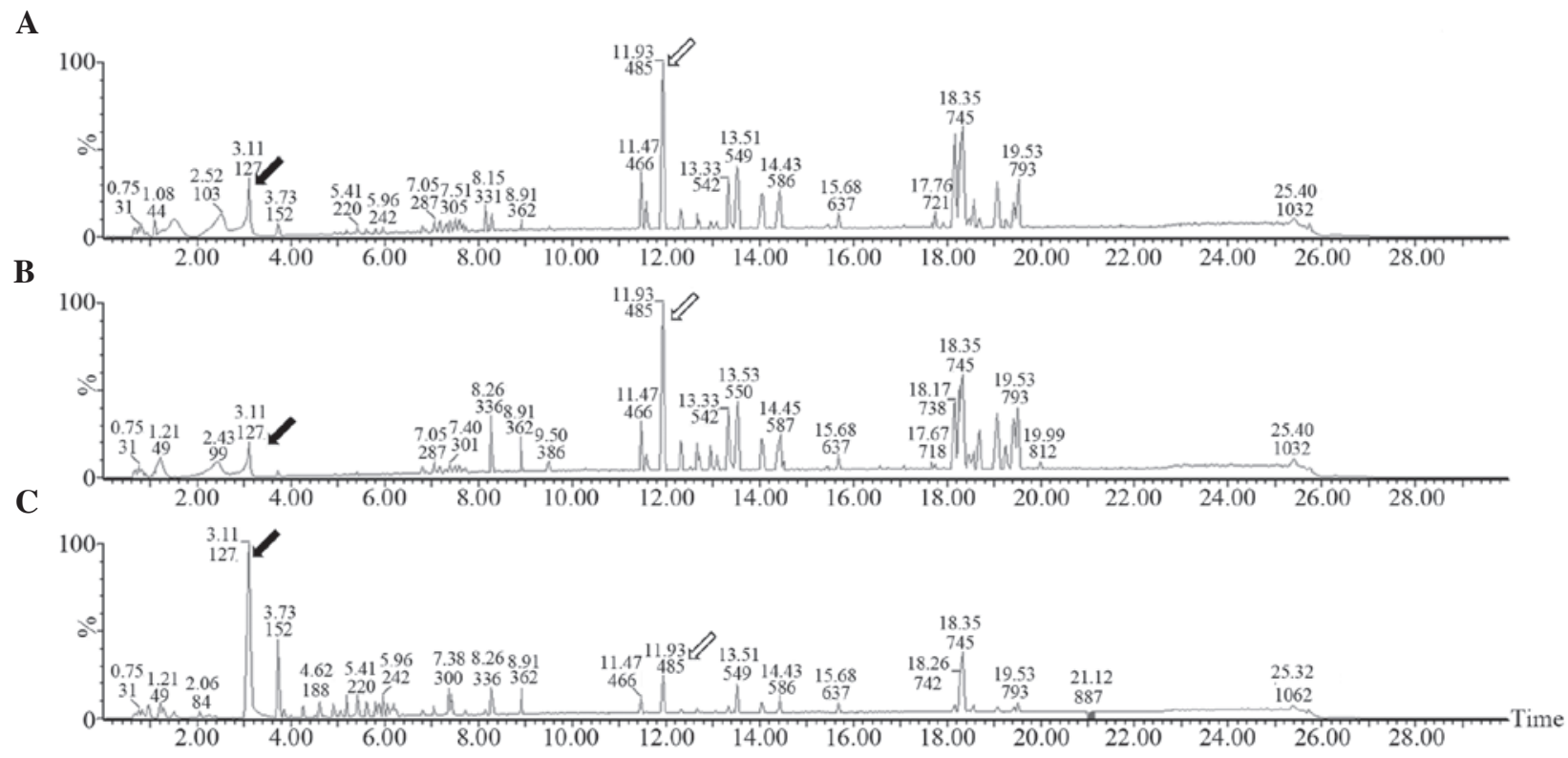

Figure 3. Typical BPI chromatograms of serum samples from patients with CRC prior to and following operation, and healthy control group. (A) BPI chromatogram of serum sample of one of the pre-operative CRC patients. (B) Representative BPI chromatogram of serum sample of the same CRC patient as in A at the time-point following surgery. (C) Representative BPI chromatogram of serum sample of one of the healthy volunteer. Among the metabolites, several were increased in CRC patients compared with those in the healthy controls (hollow arrows), while others were decreased (black arrows). BPI, base peak intensity; CRC; colorectal cancer.

those in group $3(\mathrm{P}<0.001)$. Following surgery, the CEA levels were markedly decreased by $\sim 8$-fold in group 2 (Fig. 2A). Two distinct populations with high and low levels of CEA were observed in group 1 , which may be due to the expression of CEA depending on the location of the tumor (colon or rectal). By contrast, a significant increase was noted in $\mathrm{Hb}$ levels following surgery $(\mathrm{P}<0.001)$, while no statistically significant difference was present between groups 2 
and 3 , thereby exhibiting a different pattern of alteration from that of CEA (Fig. 2B). Furthermore, the red blood cell count was slightly decreased in group 1 compared to that in groups 2 and 3, while the white blood cell count in group 1 was slightly increased compared with that in groups 2 and 3 (Fig. 2C and D), indicating that the anemia of the patients improved with surgical treatment. However, there was no statistically significant difference in the red and white blood cell count between groups 1-3 ( $\mathrm{P}>0.05)$.

UPLC-MS analysis. Low-molecular-weight metabolites in the serum of CRC patients prior to and following operation and healthy controls are presented in base peak intensity chromatograms (Fig. 3). Compared with the healthy control group, the levels of certain metabolites were increased (hollow arrow) in the CRC patients, while others were decreased (black arrow). The peak patterns in the pre-operative group were similar to those in the post-operative group, suggesting a relatively small effect of surgical operation on the patients' metabolite patterns. However, the peak patterns in the healthy control group were obviously different from those in the pre-operative and post-operative groups.

PCA and PLS-DA analysis of UPLC-MS data. In order to illustrate the differences in the metabolic profiles among the three groups, an unsupervised PCA was first used to analyze the multivariate data. In the PCA score plot, data of the control and CRC serum samples did not cluster sufficiently, as shown in Fig. 4A. Therefore, a supervised PLS-DA was performed, which was better at distinguishing the variation compared with the PCA method; it was therefore used in order to discriminate the three groups according to their the metabolic differences. The PLS-DA plot is shown in Fig. 4B and $\mathrm{C}$, where each data point represents an independent sample. In spite of certain overlaps between data points, the PLS-DA method was more suitable for clustering of the three groups, producing a distinguished classification. Similarities were observed within each group, and the three distinct clusters clearly represented the pre-operative, post-operative and healthy control groups in the PLS-DA scoring plot, suggesting the presence of significant metabolic differences between the three groups.

Candidate biomarker identification. According to the VIP values (the top $20 \mathrm{VIP}$ ) and the S-plot, 20 metabolites were selected as candidate biomarkers (Table II). In the S-plot, significantly different metabolites were identified among the pre-operative, post-operative and control groups, which may be utilized for discriminating between the three groups. The boxes indicate the candidate biomarker which are most suitable for the discrimination of groups in the S-plot (Fig. 5).

The HMDB, PubChem compound and KEGG databases were then searched to compare the MS data with chemical standards in order to identify the potential biomarkers, which are listed in Table II. Levels of three groups of biomarkers, lysophosphatidylcholines (LPCs), phosphatidylcholines (PCs) and diacylglycerols (DAGs), were significantly different between the patients with CRC and the healthy controls. Among them, PCs and LPCs which contain polyunsaturated fatty acids were decreased, whereas LPCs and

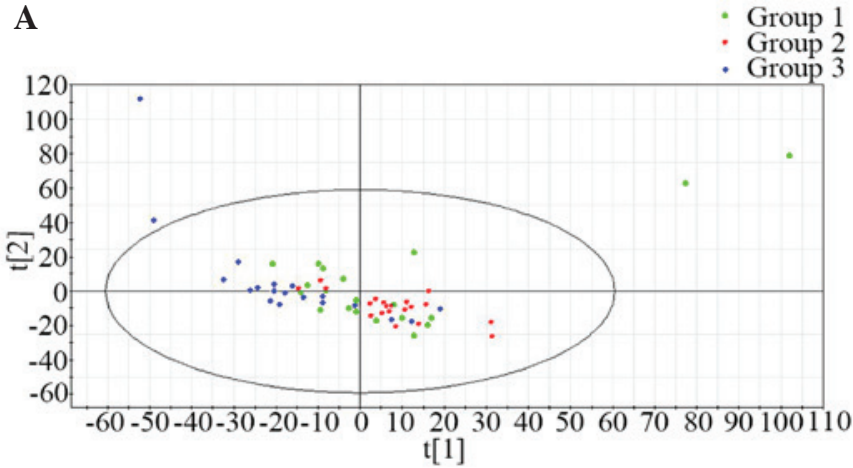

B
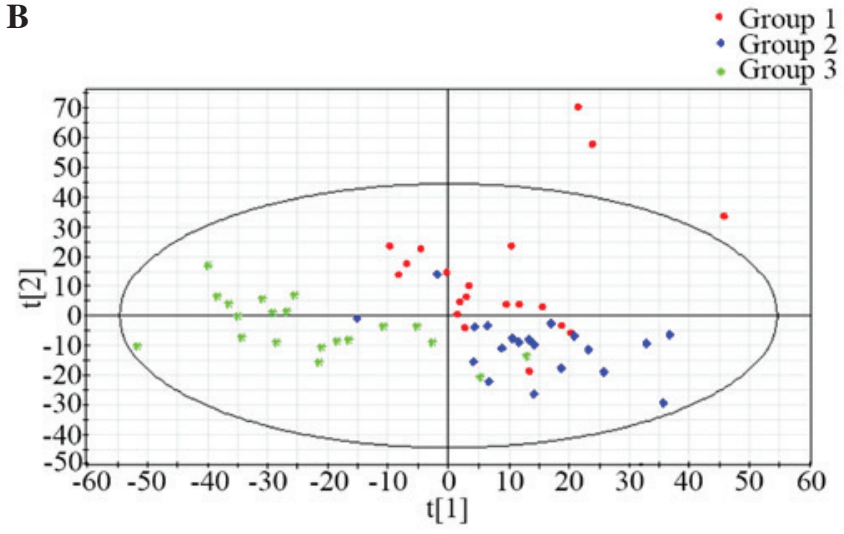

C

- Group 1

- Group 3

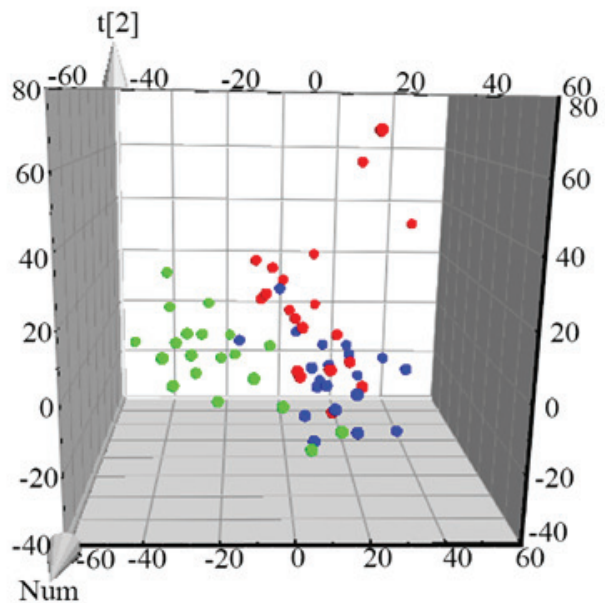

Figure 4. Unsupervised PCA and supervised PLS-DA. (A) PCA score plot in positive ion mode. Green dots represent samples of group 1, red diamonds represent samples of group 2 and blue diamonds represent samples of group 3. (B) PLS-DA score plot in positive ion mode based on UPLC-MS analysis data, showing that the three groups were clearly distinguished from each other. Red dots represent samples of group 1, blue diamonds represent samples of group 2 and green stars represent samples of group 3 . (C) PLS-DA score 3D plot in positive ion mode based on ultra-performance liquid chromatography quadrupole time-of-flight mass spectrometry data. Red represents samples of group 1, blue represents samples of group 2 and green represents samples of group 3 Group 1, patients with CRC prior to operation; group 2, patients with CRC following operation; group 3 , healthy controls. PCA, principal component analysis; PLS-DA, partial least-squares discriminant analysis; CRC, colorectal cancer; 1 , largest variation in the UPLC-MS data; t2, second-largest variation in the UPLC-MS data; Num, number; UPLC-MS, ultra-performance liquid chromatography coupled with quadrupole time-of-flight mass spectrometry.

DAGs which contain saturated fatty acids were increased in CRC patients compared with those in healthy individuals. However, the top 20 discriminating metabolites were not 
Table II. Significant serum biomarkers associated with colorectal cancer.

\begin{tabular}{|c|c|c|c|c|c|c|c|}
\hline \multirow[b]{2}{*}{ Rt } & \multirow[b]{2}{*}{$\mathrm{m} / \mathrm{z}$} & \multirow[b]{2}{*}{ VIP } & \multirow[b]{2}{*}{ Adduct } & \multirow[b]{2}{*}{ Identity } & \multicolumn{3}{|c|}{ Significance } \\
\hline & & & & & Group 1-2 & Group 1-3 & Group 2-3 \\
\hline 18.32 & 758.5718 & 10.3999 & {$[\mathrm{M}+\mathrm{H}]^{+}$} & 16:0/18:2-PC & ns & $\downarrow$ & $\downarrow \downarrow \downarrow$ \\
\hline 17.91 & 782.5719 & 9.13802 & {$[\mathrm{M}+\mathrm{H}]^{+}$} & 18:2/18:2-PC & ns & $\downarrow \downarrow \downarrow$ & $\downarrow \downarrow \downarrow$ \\
\hline 11.93 & 496.3411 & 8.91459 & {$[\mathrm{M}+\mathrm{H}]^{+}$} & LPC16:0 & ns & $\uparrow \uparrow$ & $\mathrm{ns}$ \\
\hline 19.52 & 786.6036 & 8.31944 & {$[\mathrm{M}+\mathrm{H}]^{+}$} & 18:0/18:2-PC & ns & $\downarrow$ & $\downarrow \downarrow \downarrow$ \\
\hline 18.31 & 780.5537 & 7.41249 & {$[\mathrm{M}+\mathrm{Na}]^{+}$} & 16:0/18:2-PC & ns & $\downarrow$ & $\downarrow \downarrow$ \\
\hline 19.51 & 808.5862 & 7.10026 & {$[\mathrm{M}+\mathrm{Na}]^{+}$} & 18:0/18:2-PC & ns & $\downarrow \downarrow$ & $\downarrow \downarrow \downarrow$ \\
\hline 17.92 & 804.5538 & 7.03144 & {$[\mathrm{M}+\mathrm{Na}]^{+}$} & 18:2/18:2-PC & ns & $\downarrow \downarrow \downarrow$ & $\downarrow \downarrow \downarrow$ \\
\hline 18.62 & 806.5697 & 6.58736 & {$[\mathrm{M}+\mathrm{Na}]^{+}$} & 18:1/18:2-PC & ns & $\downarrow$ & $\mathrm{ns}$ \\
\hline 11.48 & 542.3235 & 5.95541 & {$[\mathrm{M}+\mathrm{Na}]^{+}$} & LPC18:2 & ns & $\downarrow \downarrow$ & $\downarrow \downarrow$ \\
\hline 11.92 & 991.6754 & 5.70262 & {$[2 \mathrm{M}+\mathrm{H}]^{+}$} & LPC16:0 & ns & $\uparrow \uparrow \uparrow$ & $\uparrow \uparrow$ \\
\hline 18.16 & 806.5722 & 5.42741 & {$[\mathrm{M}+\mathrm{H}]^{+}$} & 16:0/22:6-PC & ns & ns & ns \\
\hline 11.48 & 520.3411 & 5.40934 & {$[\mathrm{M}+\mathrm{H}]^{+}$} & LPC18:2 & ns & $\downarrow$ & $\downarrow$ \\
\hline 11.93 & 518.3238 & 5.26510 & {$[\mathrm{M}+\mathrm{Na}]^{+}$} & LPC16:0 & ns & $\uparrow$ & ns \\
\hline 14.41 & 603.4681 & 5.11722 & {$[\mathrm{M}+\mathrm{H}]^{+}$} & DAG & ns & ns & $\uparrow \uparrow$ \\
\hline 18.62 & 784.5876 & 4.98453 & {$[\mathrm{M}+\mathrm{H}]^{+}$} & 16:0/20:3-PC & ns & ns & ns \\
\hline 13.34 & 524.3726 & 4.42997 & {$[\mathrm{M}+\mathrm{H}]^{+}$} & LPC18:0 & ns & $\uparrow$ & ns \\
\hline 11.58 & 518.3226 & 4.17220 & {$[\mathrm{M}+\mathrm{Na}]^{+}$} & LPC16:0 isomer & ns & $\uparrow$ & ns \\
\hline 18.25 & 782.5720 & 3.93874 & {$[\mathrm{M}+\mathrm{H}]^{+}$} & 16:0/20:4-PC & ns & $\downarrow$ & ns \\
\hline 19.43 & 810.6032 & 3.75328 & {$[\mathrm{M}+\mathrm{H}]^{+}$} & 18:0/20:4-PC & ns & $\downarrow$ & ns \\
\hline 17.76 & 780.5553 & 3.63848 & {$[\mathrm{M}+\mathrm{H}]^{+}$} & 16:0/20:5-PC & ns & ns & ns \\
\hline
\end{tabular}

Rt, retention time; VIP, variable importance in projection; PC, phosphatidylcholine; LPC, lysophosphatidylcholine; DAG, diacylglycerols. ns, no significant differences ( $\mathrm{P}>0.05)$; ' $\uparrow$ ' or ' $\downarrow$ ', $\mathrm{P}<0.05$; ‘ $\uparrow$ ' or ' $\downarrow \downarrow$ ', $\mathrm{P}<0.01$; ‘ $\uparrow \uparrow \uparrow$ ' or ' $\downarrow \downarrow \downarrow$ ', $\mathrm{P}<0.001$, for comparison of group 1 with group 2 , group 1 with group 3 or group 2 with group 3 .

significantly different between pre-operative and post-operative $\mathrm{CRC}$ patients $(\mathrm{P}>0.05)$; therefore, they are not suitable for discriminating between pre- and post-operative patients.

\section{Discussion}

Metabonomics is a rapidly developing discipline that provides a broad scope as well as direct information on complex cellular responses with a low requirement of material and sample preparation (14). It has been extensively applied in human diseases and has significantly contributed to the discovery of novel biomarkers of diseases. CRC represents a major cause of cancer-associated mortality worldwide (1). Metabonomics has offered a novel perspective regarding the genesis of CRC as well as an approach towards cancer diagnosis. Recently, multiple biomarkers were identified in the tissues and biofluids of CRC patients $(1,2,5)$. However, most of these markers have also been discovered in several other metabolic disorders (15-17). Therefore, specific biomarkers for monitoring CRC remain to be discovered.

The present study utilized UPLC-MC in order to detect serum metabolites in patients with CRCs. Due to the large amount of data obtained, multivariate statistical analysis models, including PCA and PLS-DA, were used to discriminate between pre-operative and post-operative groups as well as healthy controls. By using PLS-DA, in spite of certain

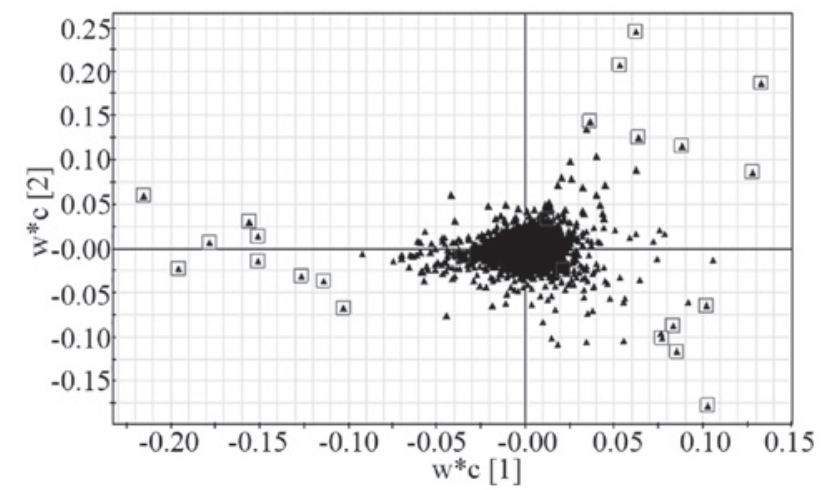

Figure 5. S-plot of partial least squares in serum samples representing the metabolites responsible for clustering results. The boxes indicate candidate biomarkers which are most suitable for the discrimination of groups in the score plot. w* c, weight of variable.

overlaps between data-points, three distinct clusters representing the three different groups were obtained. Within each group, in-group similarities were observed, which distinguished them from the other groups. Candidate biomarkers for CRC patients at different time-points of treatment were selected according to VIP values and the S-plot. Metabolites that significantly contributed to the discrimination of CRC patients were identified as LPCs, PCs and DAGs. Most of the PCs were decreased in CRC patients, while the majority of the 
LPCs and DAGs were markedly increased. Of note, LPCs which contain saturated fatty acids [e.g. LPC (16:0) and LPC (18:0)] were increased, whereas LPCs containing polyunsaturated fatty acids [e.g. LPC (18:2)] were decreased in CRC patients.

The mechanisms by which PCs decrease in the serum of CRC patients may depend on several factors. One of them may be associated with the decrease in PC synthesis. PCs are major lipid components of biomembranes, produced by two pathways: The de novo pathway (Kennedy pathway) and the re-modeling pathway (Land's pathway) (18). In the Kennedy pathway, PCs are synthesized by CDP-choline and diacylglycerol under the catalysis of PC synthase. Almost all cancer types, including CRC (19), lung cancer (20), heptocellular carcinoma (21), are characterized by specific shift in energy metabolism. A predominance of aerobic glycolysis over oxidative phosphorylation (Warburg effect) is usually present in cancer cells (22). As a result, the insufficient formation of adenosine triphosphate and CDP-choline induce a reduction in PC synthesis. Another mechanism is based on the susceptibility of lipids containing polyunsaturated fatty acids to free radicals and enzymes (23). Due to oxidative stress, the integrity of cellular membranes is destroyed and the generated lipid hydroperoxide becomes a major reaction product (24). Lipid hydroperoxide has been detected in a diverse range of diseases, including diabetes (15), cancer (16), arthritis (17) and Alzheimer's disease (25). The results of the present study showed that the PCs which were decreased in CRC patients were mainly polyunsaturated PCs. Thus, the other possible reason for the reduction of $\mathrm{PCs}$ is the peroxidation and transformation of PCs. In addition, following surgery, compared with the healthy controls, the serum levels of PCs decreased even more significantly than those in the pre-operative group, suggesting that the decreased levels of PCs following surgery may be the result of injury-induced elevation of aerobic glycolysis. This hypothesis is required to be explored by further studies.

LPCs are the hydrolysis products of PCs. The present study showed that LPCs containing polyunsaturated fatty acids were downregulated in CRC patients, which was in line with the opposite trend for PCs containing polyunsaturated fatty acids. Phospholipase A2, a critical enzyme in catalyzing the hydrolysis of PCs into LPCs, has an anti-tumorigenic role in various types of cancer (26-28), and its low activity in CRC patients may contribute to the decrease of LPCs. LPCs act as a bioactive mediator in various biological processes, including injury and inflammatory responses $(29,30)$, cellular motility, growth and regulation of differentiation (31). Numerous clinical experiments have shown that serum LPCs levels are lower in patients with advanced cancer (32-34). However, the results of the present study also showed that LPCs containing saturated fatty acids were upregulated in CRC patients. A possible explanation may be the different roles of various LPC sub-types in tumorigenesis. For example, certain sub-types of LPCs and other lysophospholipids have been demonstrated to elevate the production of multiple growth factors in breast cancer cells, including interleukin- 6 and -8 , which are regulators of neovascularization (35).

DAGs are well-known secondary messengers in signaling pathways regulating cell proliferation and apoptosis $(36,37)$. DAGs are also involved in the structural regulation of organelle morphology. Acute DAG deletion disables nuclear membrane assembly and causes alterations of endoplasmic reticulum morphology (38). In the present study, the levels of DAGs were not significantly different between the pre-operative group and the healthy control group. However, following surgery, the serum levels of DAGs were markedly increased when compared to those in the healthy controls, indicating that DAGs may function as structural components of organelles to repair cells damaged during the operation.

In conclusion, the present metabonomics study used the novel UPLC-QTOF-MS method, which is a sensitive and effective tool for biomarker discovery, to identify three groups of biomarkers for CRC, namely LPCs, PCs and DAGs. PCs and LPCs containing polyunsaturated fatty acids were decreased, whereas LPCs and DAGs containing saturated fatty acids were increased in CRC patients. To the best of our knowledge, the present study was the first to use an UPLC-QTOF-MS-based serum metabolite analysis approach to compare CRC patients at various time-points of treatment with healthy controls. Although the number of participants included in the present study was relatively small, a clear discrimination between CRC patients and healthy volunteers was observed. However, the absence of significant differences in metabolite profiles between pre-operative and post-operative groups may suggest the importance and necessity of post-operative medical procedures, such as chemotherapy. Once the serum metabonomic results of the present study are validated in a larger number of patients, it is anticipated that serum analysis using UPLC-QTOF-MS may become a standard clinical procedure to effectively diagnose and monitor CRC patients.

\section{Acknowledgements}

The present study was supported by the National Basic Research Program of China (973 Program; grant no. 2013CB531402). The authors would like to thank the State Key Laboratory for Diagnosis and Treatment of Infectious Diseases, the First Affiliated Hospital, Medical College, Zhejiang University (Hangzhou, China) for their assistance with the operation of the UPLC-QTOF-MS apparatus.

\section{References}

1. Sideris M and Papagrigoriadis S: Molecular biomarkers and classification models in the evaluation of the prognosis of colorectal cancer. Anticancer Res 34: 2061-2068, 2014.

2. Zhang A, Sun H, Yan G, Wang P, Han Y and Wang X: Metabolomics in diagnosis and biomarker discovery of colorectal cancer. Cancer Lett 345: 17-20, 2014.

3. Møller Sørensen N, Vejgaard Sørensen I, Ørnbjerg Würtz S, Schrohl AS, Dowell B, Davis G, Jarle Christensen I, Nielsen HJ and Brünner N: Biology and potential clinical implications of tissue inhibitor of metalloproteinases-1 in colorectal cancer treatment. Scand J Gastroenterol 43: 774-786, 2008.

4. Berkovich L, Shpitz B, Ghinea R, Greemland I, Kravtsov V, Kidron D, Mishaeli M and Avital S: Evaluation of peritoneal CEA levels following colorectal cancer surgery. J Surg Oncol 110: 458-462, 2014.

5. Goedert JJ, Sampson JN, Moore SC, Xiao Q, Xiong X, Hayes RB, Ahn J, Shi J and Sinha R: Fecal metabolomics: Assay performance and association with colorectal cancer. Carcinogenesis 35: 2089-2096, 2014.

6. Griffin JL and Bollard ME: Metabonomics: Its potential as a tool in toxicology for safety assessment and data integration. Curr Drug Metab 5: 389-398, 2004. 
7. Wu T, Xie G, Ni Y, Liu T, Yang M, Wei H, Jia W and Ji G: Serum metabolite signatures of type 2 diabetes mellitus complications. J Proteome Res 14: 447-456, 2015.

8. Chan AW, Gill RS, Schiller D and Sawyer MB: Potential role of metabolomics in diagnosis and surveillance of gastric cancer. World J Gastroenterol 20: 12874-12882, 2014.

9. Zhao L, Wan L, Qiu X, Li R, Liu S and Wang D: A metabonomics profiling study on phlegm syndrome and blood-stasis syndrome in coronary heart disease patients using liquid chromatography/quadrupole time-of-flight mass spectrometry. Evid Based Complement Alternat Med 2014: 385102, 2014.

10. Fanos V, Pintus MC, Lussu M, Atzori L, Noto A, Stronati M, Guimaraes H, Marcialis MA, Rocha G, Moretti C, et al: Urinary metabolomics of bronchopulmonary dysplasia (BPD): Preliminary data at birth suggest it is a congenital disease. J Matern Fetal Neonatal Med 27 (Suppl 2): 39-45, 2014.

11. Ma YL, Qin HL, Liu WJ, Peng JY, Huang L, Zhao XP and Cheng YY: Ultra-high performance liquid chromatography-mass spectrometry for the metabolomic analysis of urine in colorectal cancer. Dig Dis Sci 54: 2655-2662, 2009.

12. Wilson ID, Nicholson JK, Castro-Perez J, Granger JH, Johnson KA, Smith BW and Plumb RS: High resolution 'ultra performance' liquid chromatography coupled to oa-TOF mass spectrometry as a tool for differential metabolic pathway profiling in functional genomic studies. J Proteome Res 4: 591-598, 2005.

13. Akkoca AN, Yanık S, Ozdemir ZT, Cihan FG, Sayar S, Cincin TG, Cam A and Ozer C: TNM and modified Dukes staging along with the demographic characteristics of patients with colorectal carcinoma. Int J Clin Exp Med 7: 2828-2835, 2014.

14. Fuhrer $\mathrm{T}$ and Zamboni N: High-throughput discovery metabolomics. Curr Opin Biotechnol 31: 73-78, 2015.

15. Tsakanova GV, Ayvazyan VA, Boyajyan AS, Arakelova EA, Grigoryan GS, Guevorkyan AA and Mamikonyan AA: A comparative study of antioxidant system and intensity of lipid peroxidation in type 2 diabetes mellitus and ischemic stroke aggravated and not aggravated by type 2 diabetes mellitus. Bull Exp Biol Med 151: 564-566, 2011 .

16. Balci H, Genc H, Papila C, Can G, Papila B, Yanardag H and Uzun H: Serum lipid hydroperoxide levels and paraoxonase activity in patients with lung, breast and colorectal cancer. J Clin Lab Anal 26: 155-160, 2012.

17. Navarro-Compán V, Melguizo-Madrid E, Hernández-Cruz B, Santos-Rey K, Leyva-Prado C, González-Martín C, Navarro-Sarabia F and González-Rodríguez C: Interaction between oxidative stress and smoking is associated with an increased risk of rheumatoid arthritis: A case-control study. Rheumatology (Oxford) 52: 487-493, 2013.

18. Shindou H, Hishikawa D, Harayama T, Yuki K and Shimizu T: Recent progress on acyl CoA: Lysophospholipid acyltransferase research. J Lipid Res 50 (Suppl): S46-S51, 2009.

19. Baltaziak M, Wincewicz A, Kanczuga-Koda L, Lotowska JM, Koda M, Sulkowska U, Baltaziak M, Podbielski M, Sobaniec-Lotowska ME and Sulkowski S: The relationships between hypoxia-dependent markers: HIF-1alpha, EPO and EPOR in colorectal cancer. Folia Histochem Cytobiol 51: 320-325, 2013.

20. Zhang J, Cao J, Ma S, Dong R, Meng W, Ying M, Weng Q, Chen Z, Ma J, Fang Q, et al: Tumor hypoxia enhances non-smal cell lung cancer metastasis by selectively promoting macrophage M2 polarization through the activation of ERK signaling. Oncotarget 5: 9664-9677, 2014.

21. Lu Y, Lin N, Chen Z and Xu R: Hypoxia-induced secretion of platelet-derived growth factorBB by hepatocellular carcinoma cells increases activated hepatic stellate cell proliferation, migration and expression of vascular endothelial growth factor $\mathrm{A}$. Mol Med Rep 11: 691-697, 2015.

22. Granchi C, Fancelli D and Minutolo F: An update on therapeutic opportunities offered by cancer glycolytic metabolism. Bioorg Med Chem Lett 24: 4915-4925, 2014.
23. Catalá A: Lipid peroxidation of membrane phospholipids generates hydroxy-alkenals and oxidized phospholipids active in physiological and/or pathological conditions. Chem Phys Lipids 157: 1-11, 2009

24. Sugiyama A and Sun J: Immunochemical detection of lipid hydroperoxide- and aldehyde-modified proteins in diseases. Subcell Biochem 77: 115-125, 2014.

25. Yoo MH, Gu X, Xu XM, Kim JY, Carlson BA, Patterson AD, Cai H, Gladyshev VN and Hatfield DL: Delineating the role of glutathione peroxidase 4 in protecting cells against lipid hydroperoxide damage and in Alzheimer's disease. Antioxid Redox Signal 12: 819-827, 2009.

26. Ganesan K, Ivanova T, Wu Y, Rajasegaran V, Wu J, Lee MH, $\mathrm{Yu}$ K, Rha SY, Chung HC, Ylstra B, et al: Inhibition of gastric cancer invasion and metastasis by PLA2G2A, a novel beta-catenin/TCF target gene. Cancer Res 68: 4277-4286, 2008.

27. Fijneman RJ, Peham JR, van de Wiel MA, Meijer GA, Matise I, Velcich A and Cormier RT: Expression of Pla2g2a prevents carcinogenesis in Muc2-deficient mice. Cancer Sci 99: 2113-2119, 2008.

28. Avoranta T, Sundström J, Korkeila E, Syrjänen K, Pyrhönen S and Laine J: The expression and distribution of group IIA phospholipase A2 in human colorectal tumours. Virchows Arch 457: 659-667, 2010

29. Ma J, Yu J, Su X, Zhu C, Yang X, Sun H, Chen D, Wang Y, Cao H and Lu J: UPLC-MS-based serum metabonomics for identifying acute liver injury biomarkers in Chinese miniature pigs. Toxicol Lett 225: 358-366, 2014.

30. Nankar SA, Prajapati JS and Pande AH: Apolipoprotein E derived peptides inhibit the pro-inflammatory effect of lysophosphatidylcholine. Protein Pept Lett 21: 101-107, 2014.

31. Bassa BV, Noh JW, Ganji SH, Shin MK, Roh DD and Kamanna VS: Lysophosphatidylcholine stimulates EGF receptor activation and mesangial cell proliferation: Regulatory role of Src and PKC. Biochim Biophys Acta 1771: 1364-1371, 2007.

32. Kuliszkiewicz-Janus M, Tuz MA and Baczyński S: Application of 31P MRS to the analysis of phospholipid changes in plasma of patients with acute leukemia. Biochim Biophys Acta 1737: 11-15, 2005.

33. Taylor LA, Arends J, Hodina AK, Unger C and Massing U: Plasma lyso-phosphatidylcholine concentration is decreased in cancer patients with weight loss and activated inflammatory status. Lipids Health Dis 6: 17, 2007.

34. Süllentrop F, Moka D, Neubauer S, Haupt G, Engelmann U, Hahn J and Schicha H: 31P NMR spectroscopy of blood plasma: Determination and quantification of phospholipid classes in patients with renal cell carcinoma. NMR Biomed 15: 60-68, 2002.

35. Umezu-Goto M, Tanyi J, Lahad J, Liu S, Yu S, Lapushin R, Hasegawa Y, Lu Y, Trost R, Bevers T, et al: Lysophosphatidic acid production and action: Validated targets in cancer? J Cell Biochem 92: 1115-1140, 2004.

36. Poli A, Mongiorgi S, Cocco L and Follo MY: Protein kinase C involvement in cell cycle modulation. Biochem Soc Trans 42: 1471-1476, 2014.

37. Poli A, Ramazzotti G, Matteucci A, Manzoli L, Lonetti A, Suh PG, McCubrey JA and Cocco L: A novel DAG-dependent mechanism links PKCa and Cyclin B1 regulating cell cycle progression. Oncotarget 5: 11526-11540, 2014.

38. Domart MC, Hobday TM, Peddie CJ, Chung GH, Wang A, Yeh K, Jethwa N, Zhang Q, Wakelam MJ, Woscholski R, et al: Acute manipulation of diacylglycerol reveals roles in nuclear envelope assembly \& endoplasmic reticulum morphology. PLoS One 7: e51150, 2012 